08,04

\title{
Парамагнитные ионы в легированном европием искаженном перовските $\mathrm{YAIO}_{3}$
}

\author{
(C) В.А. Важенин ${ }^{1}$, А.П. Потапов ${ }^{1}$, А.Г. Петросян ${ }^{2}$, Г.Р. Асатрян ${ }^{3}$, А.В. Фокин ${ }^{1}$, М.Ю. Артёмов ${ }^{1}$ \\ ${ }^{1}$ Уральский федеральный университет (Институт естественных наук и математики), \\ Екатеринбург, Россия \\ ${ }^{2}$ Институт фризических исследований НАН РА, \\ Аштарак-2, Армения, \\ ${ }^{3}$ Физико-технический институт им. А.Ф. Иофрфе, \\ Санкт-Петербург, Россия \\ E-mail: Vladimir.Vazhenin@urfu.ru
}

Поступила в Редакцию 10 января 2019 г.

В окончательной редакции 15 января 2019 г.

Принята к публикации 15 января 2019 г.

В кристаллах ортоалюмината иттрия, легированных изотопом ${ }^{151} \mathrm{Eu}$, обнаружены парамагнитные центры $\mathrm{Eu}^{2+}, \mathrm{Cr}^{3+}, \mathrm{Gd}^{3+}, \mathrm{Mo}^{3+}$. Определены или уточнены параметры тонкой структуры спинового гамильтониана указанных центров. Для центров $\mathrm{Cr}^{3+}$ проведен учет ориентационного поведения ранее ненаблюдаемых запрещенных переходов. Показано, что детектируемые в магнитных полях параллельных кристаллографическим осям два сигнала $\mathrm{Mo}^{3+}$ принадлежат не разным центрам молибдена, а являются двумя внутридублетными переходами одного центра.

Работа выполнена в рамках государственного задания Минобрнауки России (3.6115.2017/8.9). Измерения проведены на спектрометре Центра коллективного пользования „Современные нанотехнологии“ Уральского федерального университета.

DOI: $10.21883 /$ FTT.2019.06.47691.353

\section{1. Введение}

Кристаллы ортоалюмината иттрия $\left(\mathrm{YAlO}_{3}\right)$, активированные ионами редких земель и группы железа, находят многочисленные применения в лазерной физике и оптоэлектронике. Для оптимизации используемых в практических приложениях характеристик этих материалов необходима информация о природе, структуре и количестве дефектов в реальных кристаллах. Усилия лабораторий, занимающихся ростом монокристаллов алюмината иттрия, направлены на получение образцов с заданным содержанием активатора и минимизацией концентрации неконтролируемых примесей. Для кристаллов, выращенных в молибденовом контейнере, неконтролируемой примесью часто являются ионы молибдена.

Спектр электронного парамагнитного резонанса (ЭПР) ионов $\mathrm{Mo}^{3+}$ (один внутридублетный переход центров молибдена $\left(4 d^{3}\right)$ с электронным спином $S=3 / 2$ ), замещающих в алюминате иттрия позиции алюминия, впервые был зарегистрирован авторами [1]. Большое начальное расщепление двух крамерсовых дублетов не позволяет детектировать междублетные переходы в $X$-диапазоне. Отнесение наблюдаемого сигнала к центру $\mathrm{Mo}^{3+}$ в [1] оказалось возможным благодаря характерной сверхтонкой структуре (СТС) этого иона, обусловленной ядерным спином $I=5 / 2$. В работе [2], кроме упомянутого выше центра молибдена [1], наблюдался внутридублетный сигнал второго центра $\mathrm{Mo}^{3+}$. Авторы [2] предполагают, что этот центр обусловлен на- личием вблизи иона молибдена какого-то дефекта. Ориентационное поведение сигналов двух центров $\mathrm{Mo}^{3+}$ в трех кристаллографических плоскостях [2] было описано ромбическим спиновым гамильтонианом с эффективным спином $S=1 / 2$.

В работе [3] нами в алюминате иттрия была исследована тонкая структура ЭПР спектра высокоспиновых $(S=7 / 2)$ центров $\mathrm{Eu}^{2+}, \mathrm{Gd}^{3+}$ и определены ее параметры. Поскольку исследованный кристалл был легирован европием с естественным содержанием изотопов и демонстрировал сложную слабо разрешенную СТС, в нем удалось оценить только параметр сверхтонкого взаимодействия $A=-95 \mathrm{MHz}$ для ${ }^{151} \mathrm{Eu}^{2+}$. Вновь выращенный кристалл, исследованный в настоящей работе, был активирован европием, обогащенным ионами одного изотопа $\mathrm{Eu}^{2+}$. В этом кристалле детектировались интенсивные спектры $\mathrm{Cr}^{3+}[4-6], \mathrm{Eu}^{2+}[3], \mathrm{Gd}^{3+}$ [3,7-9] и $\mathrm{Mo}^{3+}$, исследованные авторами [2], а также не идентифицированные узкие линии.

Данная работа посвящена идентификации наблюдаемых ЭПР-сигналов, определению или уточнению параметров тонкой структуры указанных центров, а также установлению их локализации.

\section{2. Образцы и методика эксперимента}

Несмотря на малую температурную область устойчивости фазы $\mathrm{YAlO}_{3}$ [10], получение монокристаллов 
алюмината иттрия возможно с использованием расплавных методов кристаллизации [11]. В настоящей работе выращивание монокристаллов $\mathrm{YAlO}_{3}:{ }^{151} \mathrm{Eu}$ проводилось методом вертикальной направленной кристаллизации [12,13] с использованием высокочистого оксида иттрия (марки ИтО-В), кристаллического сапфира $(99.95 \%)$ и оксида изотопа европия ${ }^{151} \mathrm{Eu}_{2} \mathrm{O}_{3}$ $\left(97.5 \%{ }^{151} \mathrm{Eu}\right.$ и $\left.2.5 \%{ }^{153} \mathrm{Eu}\right)$. Для стабилизации в решетке достаточных количеств центров $\mathrm{Eu}^{2+}$ в расплавы дополнительно вводили оксид $\mathrm{SiO}_{2}$. Состав исходных расплавов соответствовал $\mathrm{Y}_{1-x} \mathrm{Eu}_{x} \mathrm{Al}_{1-y} \mathrm{Si}_{y} \mathrm{O}_{3}(x=0.02$; $y=0.04)$. Процесс выращивания проводили в молибденовых трубках диаметром $12 \mathrm{~mm}$ на затравки, ориентированные вдоль оси $\mathbf{b}$, в атмосфере $\mathrm{Ar} / \mathrm{H}_{2}$, при скоростях $2 \mathrm{~mm} / \mathrm{h}$ и тепловых полях, обеспечивающих низкую вероятность образования микротрещин и двойников.

Качество кристаллов контролировали на поляризационном микроскопе МПС-2. Полученные кристаллы бесцветны и не окрашиваются при длительном воздействии дневного света. Наличие в кристаллах ионов европия $\left(\mathrm{Eu}^{3+}\right.$ и $\left.\mathrm{Eu}^{2+}\right)$ контролировали по спектрам поглощения на спектрофотометре SPECORD200 PLUS (по $f-f$-переходам ионов $\mathrm{Eu}^{3+}$ и полосе переноса заряда $\left.\mathrm{Eu}^{3+}-\mathrm{O}^{2-}\right)$. Примесные ионы европия замещают в решетке узлы $\mathrm{Y}^{3+}$, окруженные восьмью кислородами; ввиду разницы в ионных радиусах $\left(\mathrm{Eu}^{3+}-1.066 \AA\right.$, $\left.\mathrm{Eu}^{2+}-1.25 \AA, \mathrm{Y}^{3+}-1.019 \AA\right)$ коэффициент распределения меньше единицы и концентрация примеси по длине кристалла возрастает.

Измерения спектров ЭПР образцов $\mathrm{YAlO}_{3}:{ }^{151} \mathrm{Eu}$ проводились при комнатной температуре на спектрометре трехсантиметрового диапазона EMX Plus Bruker. Ориентация образцов в резонаторе спектрометра производилась с помощью штатного одноосного автоматического гониометра и приспособления, позволяющего вращать образец вокруг оси, перпендикулярной оси гониометра. Для достижения выделенных ориентаций (B $\| \mathbf{a}, \mathbf{b}, \mathbf{c}$, где $\mathbf{B}$ - индукция магнитного поля и $\mathbf{a}, \mathbf{b}, \mathbf{c}-$ кристаллографические оси) использовалось ориентационное поведение положений переходов $\mathrm{Mo}^{3+}, \mathrm{Cr}^{3+}$ и наконец, $\mathrm{Gd}^{3+}$, имеющих наиболее сильные угловые зависимости.

\section{3. Результаты эксперимента и их обсуждение}

Кристаллы $\mathrm{YAlO}_{3}$ имеют структуру искаженного перовскита (YАР) с пространственной группой $P b n m\left(D_{2 h}^{16}\right)[14]$ и параметрами элементарной ячейки: $a=5.176 \AA, b=5.332 \AA, c=7.356 \AA$ (для описания структуры используется и система Pnтa [15]). Центры молибдена и хрома, замещая ионы $\mathrm{Al}^{3+}$, имеют магнитную кратность 4 (группа симметрии позиции $\overline{1}(c))$. Они переходят друг в друга при отражении в плоскостях, нормальных к осям $\mathbf{a}, \mathbf{b}, \mathbf{c}$, вследствие чего в указанных плоскостях две пары центров $\mathrm{Mo}^{3+}$ и $\mathrm{Cr}^{3+}$ становятся эквивалентными, при $\mathbf{B} \| \mathbf{a}, \mathbf{b}, \mathbf{c}$ сигналы четырех центров сливаются.

Примесные редкоземельные ионы $\mathrm{Gd}^{3+}$ и $\mathrm{Eu}^{2+}$ (электронные спины $S=7 / 2$ ) в ҮАР замещают „квазиредкоземельные“ ионы $\mathrm{Y}^{3+}$, имеющие единственную операцию симметрии - отражение в плоскости ab (группа точечной симметрии $\left.-C_{S}\right)$. Четыре позиции иттрия попарно связаны операцией инверсии и отражением в плоскостях ca и cb. В случае нелокальной зарядовой компенсации примесного иона $\mathrm{Eu}^{2+}$, а также для иона $\mathrm{Gd}^{3+}$ будет существовать два магнитно-неэквивалентных центра, которые становятся эквивалентными только в этих плоскостях.

На рис. 1 приведены ЭПР-спектры алюмината иттрия с примесью $\mathrm{Eu}^{2+}$ при комнатной температуре и B $\| \mathbf{a}, \mathbf{b}, \mathbf{c}$. Как и следовало ожидать, в спектре наблюдаются довольно интенсивные группы сигналов, несомненно, принадлежащие центрам ${ }^{151} \mathrm{Eu}^{2+}(I=5 / 2)$, хотя электронных переходов с классической сверхтонкой структурой за счет взаимодействия с ядерным спином (шесть эквидистантных компонент одинаковой интенсивности) крайне мало. Это всего два перехода при В $\| \mathbf{c}$ в полях 395 тТ и $444 \mathrm{mT}$ (рис. 1).

Наиболее интенсивными в спектре оказались сигналы триклинных центров $\mathrm{Cr}^{3+}$ (рис. 1), исследованных в работах [4-6]. Причиной неожиданно высокой концентрации хрома в кристалле скорее всего являются молибденовые трубки низкого качества, использованные при выращивании образцов. Важно отметить, что сверхтонкая структура, обусловленная изотопом ${ }^{53} \mathrm{Cr}^{3+}$ $(I=3 / 2$, естественная распространенность 9.5\%), демонстрирует интенсивные запрещенные переходы с переворотом ядерного спина. Расчеты показали, что такая СТС имеет место даже при изотропном сверхтонком взаимодействии и в ориентациях магнитного поля вдоль кристаллографических осей.

В нижних частях рис. 1 приведены расчетные положения и интегральные интенсивности переходов $\mathrm{Gd}^{3+}$ и $\mathrm{Eu}^{2+}$ в YАР. Для расчета спектра $\mathrm{Eu}^{2+}$ использовались параметры моноклинного спинового гамильтониана [3] в определении [16],

$$
\begin{aligned}
H_{s p}= & g \beta(\mathbf{B S})+1 / 3\left(b_{20} O_{20}+b_{21} O_{21}+b_{22} O_{22}\right) \\
& +1 / 60\left(b_{40} O_{40}+b_{41} O_{41}+b_{42} O_{42}+b_{43} O_{43}\right. \\
& \left.+b_{44} O_{44}\right)+1 / 1260 \sum_{m} b_{6 m} O_{6 m},
\end{aligned}
$$

где $g-g$-фактор, $\beta$ - магнетон Бора, $O_{n m}-$ спиновые операторы Стивенса [16], $b_{n m}$ - параметры тонкой структуры. Величины параметров в [3] получены с использованием примерных положений центров СТС в системе координат X $\|\mathbf{a}, \mathrm{Y}\| \mathbf{c}, \mathrm{Z} \| \mathbf{b}(\mathbf{c}-$ нормаль к плоскости отражения в группе симметрии позиции). Как видно, многие переходы $\mathrm{Eu}^{2+}$ перекрываются как друг с другом, так и с сигналами $\mathrm{Gd}^{3+}$ или $\mathrm{Cr}^{3+}$, что сильно 
затрудняет анализ достаточно сложной СТС. Рассмотрение в алюминате иттрия сверхтонкой структуры центров ${ }^{151} \mathrm{Eu}^{2+}$, а также ионов ${ }^{95} \mathrm{Mo}^{3+},{ }^{97} \mathrm{Mo}^{3+}{ }_{\text {и }}^{53} \mathrm{Cr}^{3+}$ будет предметом следующей публикации.

Положения и интенсивности резонансов $\mathrm{Gd}^{3+}$ рассчитаны с параметрами табл. 1, которые являются резуль-
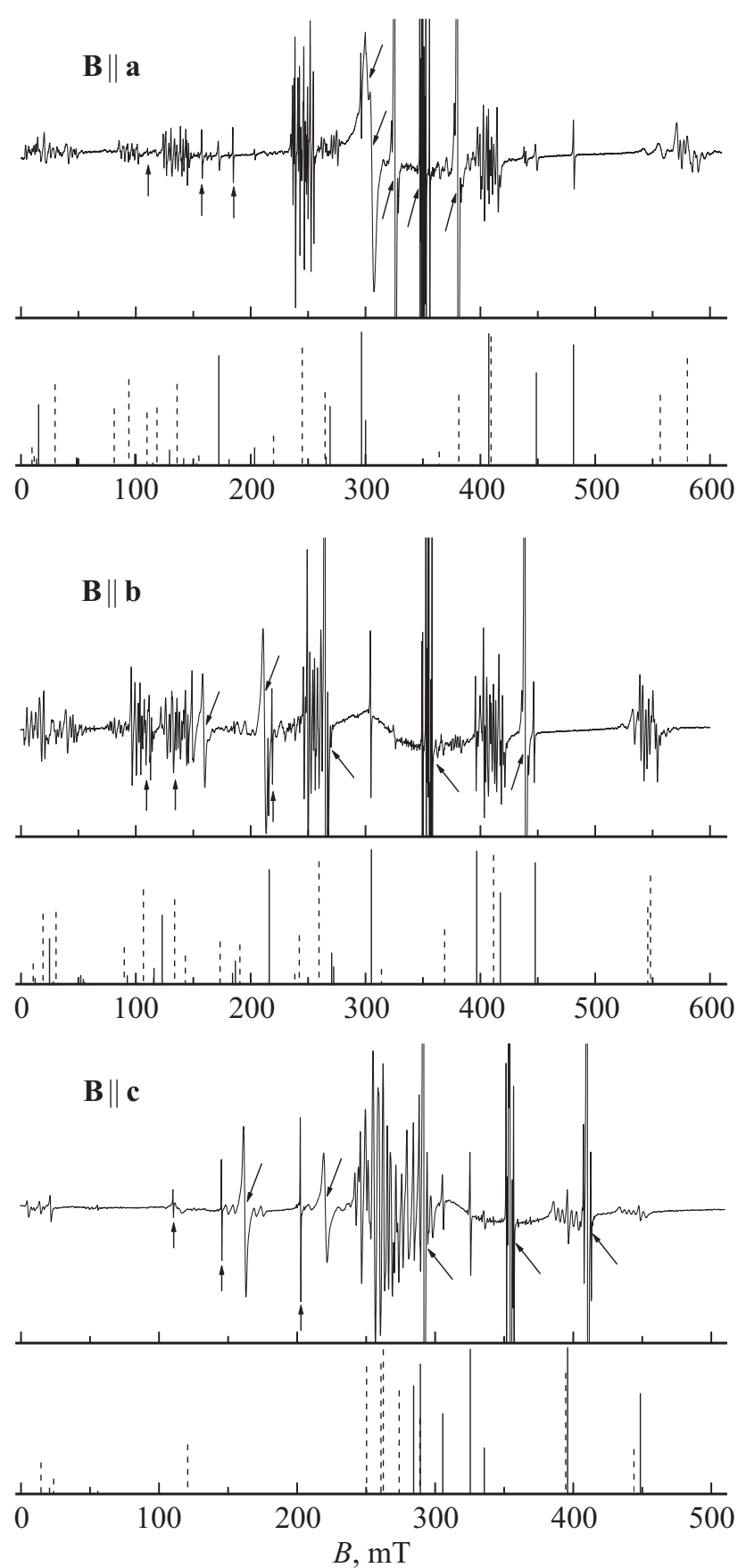

Рис. 1. ЭПР-спектр $\mathrm{YAlO}_{3}:{ }^{151} \mathrm{Eu}^{2+}$ при $\mathbf{B} \| \mathbf{a}, \mathbf{b}, \mathbf{c}$ и комнатной температуре на частоте $9833 \mathrm{MHz}$. В нижней части рисунков приведены результаты расчета положений и интегральных интенсивностей переходов: сплошные отрезки - для $\mathrm{Gd}^{3+}$, штриховые - для центров сверхтонкой структуры переходов $\mathrm{Eu}^{2+}$. Верхние стрелки указывают переходы ионов $\mathrm{Mo}^{3+}$, нижние - переходы центров $\mathrm{Cr}^{3+}$.
Таблица 1. Параметры спинового гамильтониана центров $\mathrm{Gd}^{3+}$ в $\mathrm{YAlO}_{3}$ в системе координат: $\mathbf{x}\|\mathbf{a}, \mathbf{y}\| \mathbf{b}, \mathbf{z} \| \mathbf{c}$. Среднеквадратичное отклонение расчетных частот от экспериментальных $F(N)$ (где $N$ - число использованных экспериментальных положений сигналов) и параметры $b_{n m}, c_{n m}$ приведены в $\mathrm{MHz}$

\begin{tabular}{c|c|c}
\hline Параметры & {$[8,9]$} & Данная работа \\
\hline$g_{x}$ & 1.9918 & 1.991 \\
$g_{y}$ & 1.9935 & 1.991 \\
$g_{z}$ & 1.9929 & 1.991 \\
$b_{20}$ & $-334.8^{*}$ & $-333.5^{*}$ \\
$b_{22}$ & 1621.4 & 1619.5 \\
$c_{22}$ & 2432.2 & 2425.7 \\
$b_{40}$ & 19.0 & 19.2 \\
$b_{42}$ & -69.0 & -65.2 \\
$c_{42}$ & -170.1 & -167.5 \\
$b_{44}$ & -123.3 & -125.1 \\
$c_{44}$ & 99.9 & 95.6 \\
$b_{60}$ & & $-1.6^{* *}$ \\
$F(N)$ & & $7(23)$
\end{tabular}

Примеч ан ие. ${ }^{*}$ знак $b_{20}$ не определялся, ${ }^{* *}$ остальные параметры шестого ранга малы.

татом оптимизации параметров моноклинного спинового гамильтониана [16] методом наименьших квадратов на множестве экспериментальных положений переходов рис. 1.

Следует отметить хорошее согласие полученных нами параметров (табл. 1) с результатами авторов $[8,9]$. Заметное отличие параметров табл. 1 от значений, приведенных в нашей предыдущей работе [3], объясняется меньшим количеством переходов, использованных в [3] в процедуре fitting. Этот факт обусловлен малой интенсивностью спектра гадолиния, например, при В $\|$ с не было зарегистрировано ни одного перехода центров $\mathrm{Gd}^{3+}$.

Как видно на рис. 1, сигнал, отнесенный авторами [2] к центру $\mathrm{Mo}^{3+}$, при В $\|$ с демонстрирует шесть компонент СТC, две из которых находятся под интенсивным переходом четных изотопов. Это хорошо согласуется с наличием у молибдена двух изотопов: ${ }^{95} \mathrm{Mo}(I=5 / 2$, распространенность $15.7 \%)$ и ${ }^{97} \mathrm{Mo}(I=5 / 2$, распространенность 9.5\%) с почти равными значениями ядерного магнитного момента. Для этого перехода величина параметра сверхтонкого взаимодействия $A \approx 137 \mathrm{MHz}$, что близко к значениям $A \approx 130 \mathrm{MHz}$ и $A \approx 143 \mathrm{MHz}$, полученным в работе [17] для тригональных центров $\mathrm{Mo}^{3+}$ в гранатах $\mathrm{Y}_{3} \mathrm{Al}_{5} \mathrm{O}_{12}$ и $\mathrm{Lu}_{3} \mathrm{Al}_{5} \mathrm{O}_{12}$ соответственно.

Предположение [2] о том, что второй центр $\mathrm{Mo}^{3+}$ (второй сигнал молибдена на рис. 1) обусловлен ионом молибдена в позиции $\mathrm{Al}^{3+}$, ассоциированным с неким дефектом, не кажется правдоподобным. Поскольку зарядовые состояния примесного и матричного ионов одинаковы, нет разумных механизмов ассоциации $\mathrm{Mo}^{3+}$ с дефектами.

$\mathrm{B}$ решетке $\mathrm{YAlO}_{3}$ имеется две позиции с точечной симметрией $\overline{1}\left(C_{i}\right)[18,19]$, только в одной из которых 


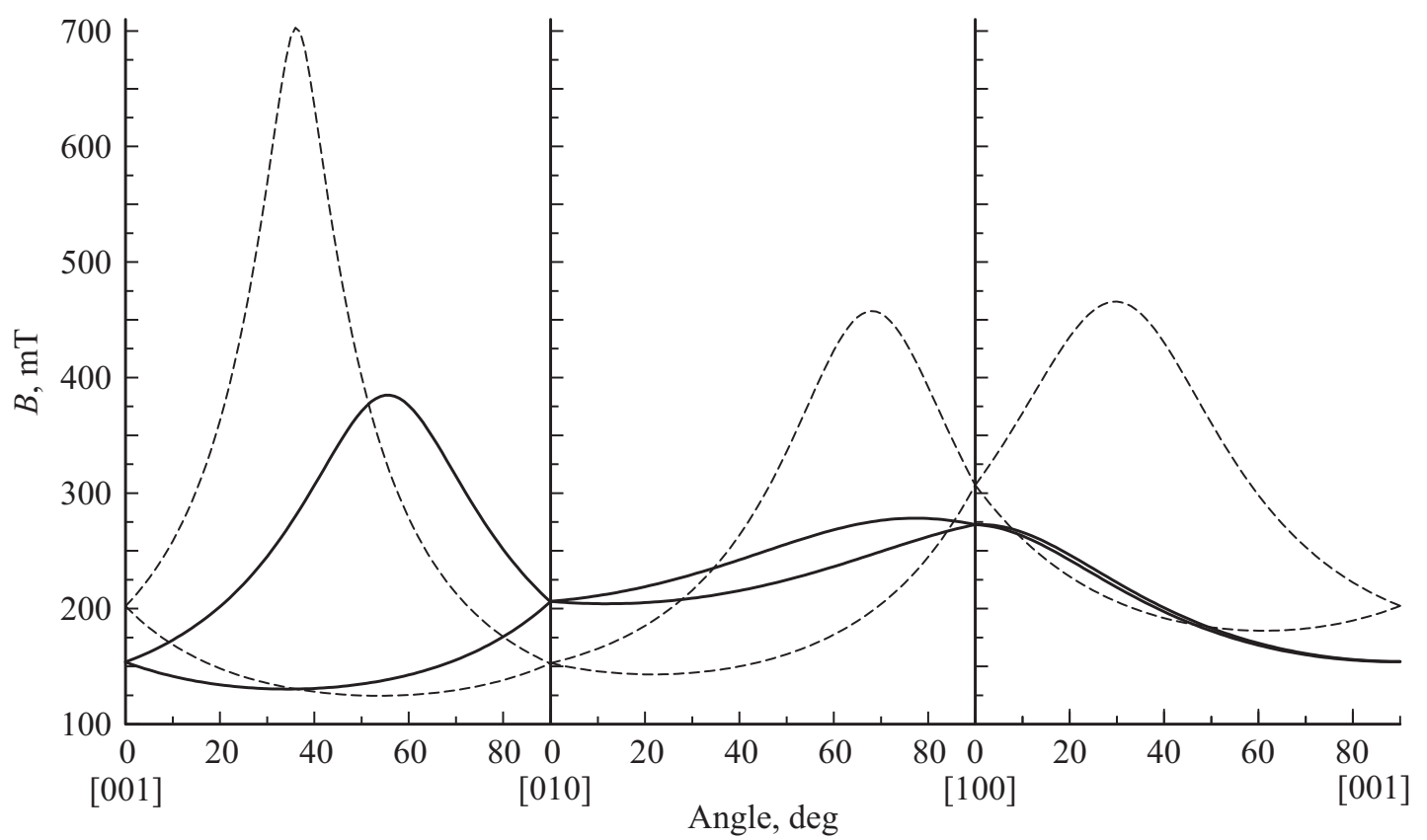

Рис. 2. Ориентационное поведение положений переходов $1 \leftrightarrow 2$ (сплошные кривые) и $3 \leftrightarrow 4$ (штриховые) центров Мо ${ }^{3+}$, полученное в результате расчета с параметрами первой колонки табл. 2.

располагается ион алюминия, окруженный шестью ионами кислорода на расстоянии $\approx 0.19 \mathrm{~nm}$, вторая сфера состоит из шести ионов иттрия с расстоянием до них больше $0.3 \mathrm{~nm}$. В ближайшем окружении второй позиции имеется три пары ионов кислорода с расстояниями $0.157,0.219$ и $0.284 \mathrm{~nm}$ и два иона $\mathrm{Y}^{3+}$, располагающихся на расстоянии $0.186 \mathrm{~nm}$, что говорит о крайней энергетической невыгодности помещения в нее $\mathrm{Mo}^{3+}$ или $\mathrm{Al}^{3+}$.

В связи с отсутствием веских аргументов в пользу существования альтернативной локализации $\mathrm{Mo}^{3+}$ в решетке $\mathrm{YAlO}_{3}$, было выдвинуто предположение о регистрации в [2] переходов не двух центров $\mathrm{Mo}^{3+}$, а двух внутридублетных переходов одного центра. Для центра со спином $S=3 / 2$ и большим начальным расщеплением в случае локализации примесного иона в позиции с достаточно низкой точечной симметрией оба внутридублетных перехода соизмеримой интенсивности могут наблюдаться во всем диапазоне углов. Такая ситуация имела место для центров $\mathrm{Cr}^{3+}$ в моноклинных кристаллах $\mathrm{Y}_{2} \mathrm{SiO}_{5}$ и $\left.\mathrm{Sc}_{2} \mathrm{SiO}_{5}[20]\right)$.

В связи с этим, была предпринята попытка описать зависимости рис. 2 работы [2] спиновым гамильтонианом вида [16]

$$
\begin{aligned}
H_{s p}= & \beta(\mathbf{B g S})+1 / 3\left(b_{20} O_{20}+b_{21} O_{21}\right. \\
& \left.+b_{22} O_{22}+c_{21} \Omega_{21}+c_{22} \Omega_{22}\right),
\end{aligned}
$$

где $O_{n m}$ и $\Omega_{n m}$ - косинусоидальные и синусоидальные спиновые операторы Стивенса, с $S=3 / 2$ в предположении, что авторами [2] измерено поведение внутридублетных переходов $(1 \leftrightarrow 2$ и $3 \leftrightarrow 4)$ одного центра. Параметры (2), полученные в процедуре оптимизации, приведены в первой колонке табл. 2, а описываемые ими угловые зависимости положений сигналов $\mathrm{Mo}^{3+}$ из [2] показаны на рис. 2.

Так как данные для оптимизации параметров (2) были получены оцифровкой рис. 2 из [2], среднеквадратичное отклонение экспериментальных и расчетных положений сигналов $\mathrm{Mo}^{3+}$ в трех кристаллографических плоскостях достаточно велико. Но сравнение рассчитанных зависимостей и приведенных на рис. 2 в [2] показывает, что описание сигналов $\mathrm{Mo}^{3+}$ спином $S=3 / 2$ повторяет все наблюдаемые особенности в ориентационном поведении центров $\mathrm{Mo}^{3+}[2]$. Кроме того, оказалось, что полученные параметры предсказывают в плоскости bс в небольшом диапазоне углов, в полях $\approx 1500 \mathrm{mT}$ существование еще одного, не наблюдавшегося в [2], перехода $3 \leftrightarrow 4$.

Таблица 2. Параметры триклинного спинового гамильтониана центров $\mathrm{Mo}^{3+}$ в $\mathrm{YAlO}_{3}$ в системе координат: $\mathbf{x}\|\mathbf{a}, \mathbf{y}\| \mathbf{b}$, $\mathbf{z} \| \mathbf{c}\left(b_{n m}, c_{n m}\right.$ и $F(N)$ приведены в $\left.\mathrm{MHz}\right)$

\begin{tabular}{c|c|c}
\hline Параметры & Данные [2], 20 K & Данная работа, 300 K \\
\hline$g_{x}$ & 1.961 & 1.970 \\
$g_{y}$ & 1.916 & 1.975 \\
$g_{z}$ & 1.982 & 1.970 \\
$b_{20}$ & $-8400^{*}$ & $-10350^{*}$ \\
$b_{21}$ & -22150 & -23030 \\
$b_{22}$ & -11200 & -9940 \\
$c_{21}$ & 104450 & 113560 \\
$c_{22}$ & -17400 & -20560 \\
$F(N)$ & $250(190)$ & $32(162)$
\end{tabular}

Примечан ие. * знак $b_{20}$ не определялся. 
Таблица 3. Соотношение знаков параметров спинового гамильтониана (2) четырех центров, связанных отражением в плоскостях $\sigma$

\begin{tabular}{c|c|c|c|c}
\hline \multirow{2}{*}{ Параметры } & 1 & 2 & 3 & 4 \\
& исходный & $\sigma \perp \mathbf{z}$ & $\sigma \perp \mathbf{y}$ & $\sigma \perp \mathbf{x}$ \\
\hline$b_{20}$ & + & + & + & + \\
$b_{21}$ & + & - & + & - \\
$b_{22}$ & + & + & + & + \\
$c_{21}$ & + & - & - & + \\
$c_{22}$ & + & + & - & -
\end{tabular}

Уровни энергии центров $\mathrm{Mo}^{3+}(S=3 / 2)$ в этом случае $\left(\theta=34.5^{\circ}, \varphi=90^{\circ}\right)$ приведены на рис. 3 .

Для подтверждения результатов расчета, полученных с использованием экспериментальных данных работы [2], нами были выполнены измерения ориентационного поведения положений сигналов $\mathrm{Mo}^{3+}$ в плоскости bc. Образцы не были предварительно ориентированы, поэтому измерение угловой зависимости положений переходов проводилось от „точной“ ориентации В $\|$ с до кристаллографической плоскости ab. Результаты приведены на рис. 4, отсутствие экспериментальных точек в районе $300-400 \mathrm{mT}$ объясняется невозможностью определения положений переходов $\mathrm{Mo}^{3+}$ из-за интенсивных сигналов $\mathrm{Cr}^{3+}$ и $\mathrm{Eu}^{2+}$.

Расщепление всех кривых на рис. 4 обусловлено небольшим отклонением магнитного поля от плоскости bc. Величину выхода поля из плоскости около b $\left(\approx 3^{\circ}\right)$ удалось оценить, моделируя расщепление одного из интенсивных и хорошо измеряемых переходов центров $\mathrm{Gd}^{3+}$ (см. рис. 5) с использованием параметров табл. 1.

Обнаружение высокополевого перехода $3 \leftrightarrow 4$ в полях $\approx 1500 \mathrm{mT}$ (рис. 4), а также качественное описание положений переходов $\mathrm{Mo}^{3+}$ в ориентациях магнитного поля вдоль кристаллографических осей и вблизи плоскости bс (рис. 1 и 4), позволяют сделать однозначный вывод о существовании в ортоалюминате иттрия лишь одного центра $\mathrm{Mo}^{3+}$, локализованного в позиции $\mathrm{Al}^{3+}$. Параметры гамильтониана (2) этого центра приведены в табл. 2 (вторая колонка). Спектры четырех одинаковых, но различно ориентированных центров $\mathrm{Mo}^{3+}$ (а также любого другого парамагнитного иона, локализованного в той же позиции) в произвольной ориентации магнитного поля могут быть рассчитаны при изменении знаков параметров спинового гамильтониана согласно табл. 3 [21].

Для описания СТС ионов ${ }^{53} \mathrm{Cr}^{3+}(S=3 / 2, I=3 / 2)$ необходимо знание параметров спинового гамильтониана (2), описывающих тонкую структуру спектра ЭПР этого центра. Для изотопов хрома с нулевым ядерным спином эти параметры приводятся в целом ряде работ [4-6], но, к сожалению, в разных системах координат. Наличие в ориентациях $\mathbf{B} \| \mathbf{a}, \mathbf{b}, \mathbf{c}$ (рис. 1) легко идентифицируемых интенсивных сигналов $\mathrm{Cr}^{3+}$,

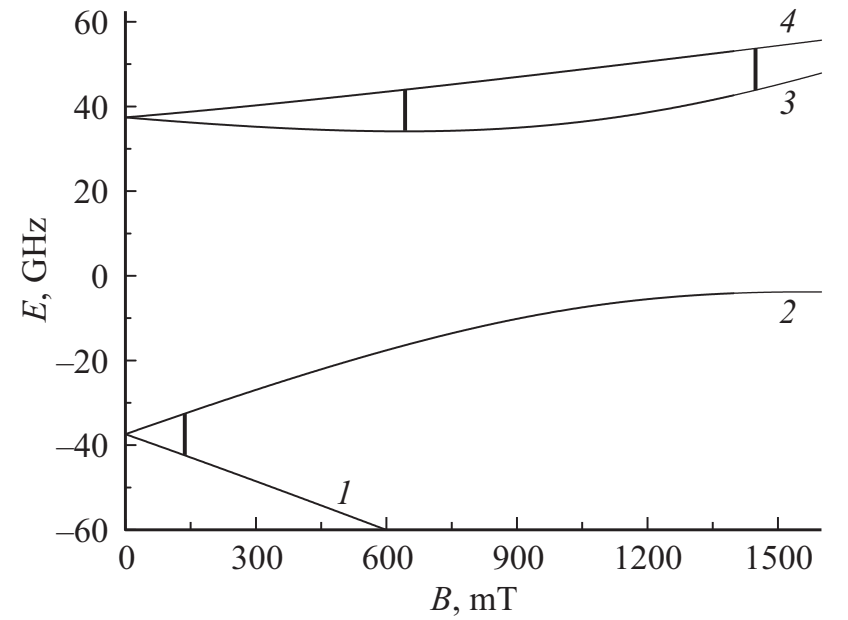

Рис. 3. Уровни энергии центров $\mathrm{Mo}^{3+}$ при $\theta=34.5^{\circ}$ и $\varphi=90^{\circ}$.

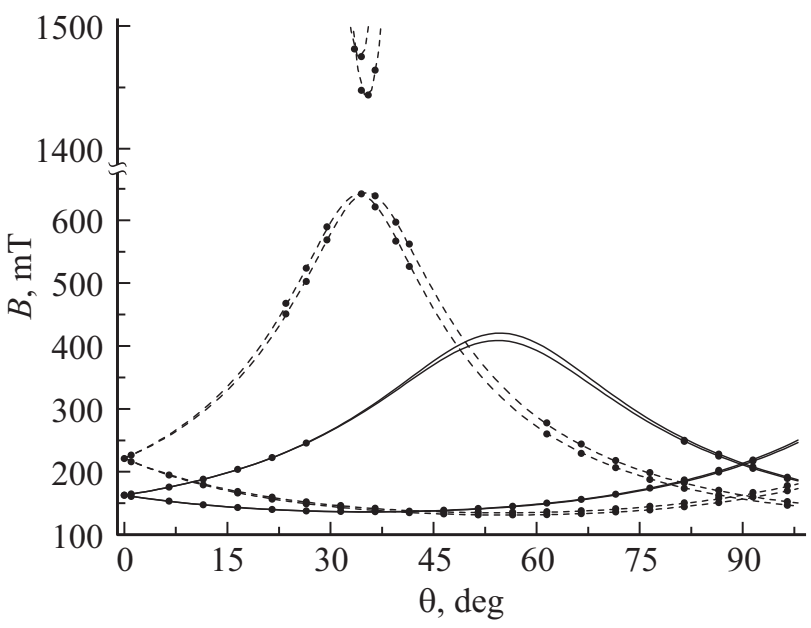

Рис. 4. Ориентационное поведение центров $\mathrm{Mo}^{3+}$ при вращении магнитного поля вблизи плоскости $\mathbf{z y}(\mathbf{c b})$, точки эксперимент, кривые - расчет при $\varphi=90 \pm 3^{\circ}$ (сплошные кривые - переход $1 \leftrightarrow 2$, штриховые - переход $3 \leftrightarrow 4)$.

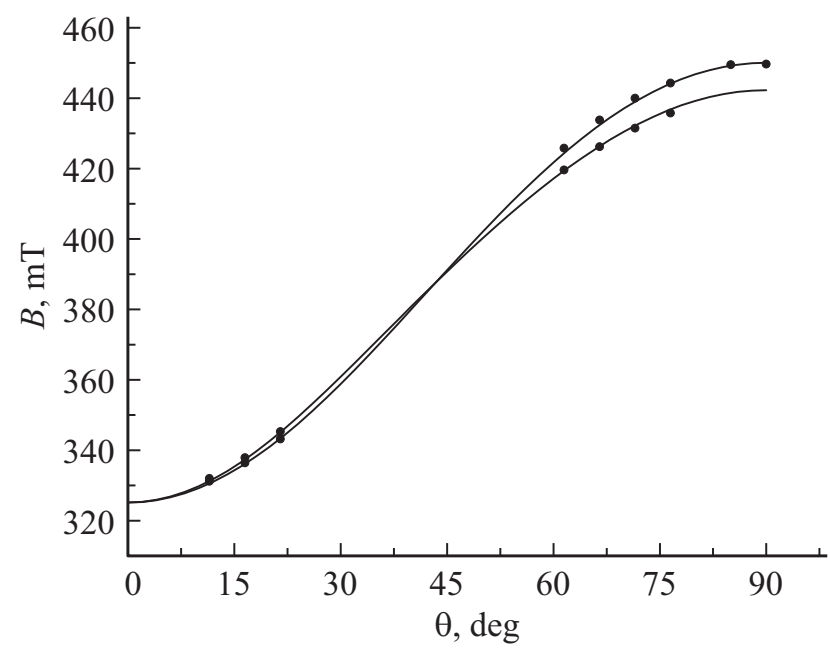

Рис. 5. Полярная угловая зависимость перехода $5 \leftrightarrow 6$ центров $\mathrm{Gd}^{3+}$ вблизи плоскости $\mathbf{z y}(\mathbf{c b})$, точки - эксперимент, кривые - расчет при $\varphi=90 \pm 3^{\circ}$. 
а также регистрации их ориентационного поведения вблизи плоскости bc (рис. 6), позволили определить для ионов хрома параметры триклинного гамильтониана (2) в системе координат $\mathbf{x}\|\mathbf{a}, \mathbf{y}\| \mathbf{b}, \mathbf{z} \| \mathbf{c}$. Как видно (рис. 6), полученные параметры хорошо описывают в диапазоне (250-450) $\mathrm{mT}$ полярную угловую зависимость положений трех разрешенных переходов $(1 \leftrightarrow 2$, $2 \leftrightarrow 3$ и $3 \leftrightarrow 4$ ) четырех магнитно неэквивалентных центров $\mathrm{Cr}^{3+}$ при учете выхода магнитного поля из плоскости bс около $\mathbf{b}$ на угол $\approx 3^{\circ}$.

Ориентационное поведение вблизи плоскости bc упомянутых во введении не идентифицированных интенсивных и узких сигналов, помеченных на рис. 1 вертикальными стрелками, показано на рис. 6 штриховыми кривыми. Видно, что магнитная кратность этих сигналов также равна 4, и, следовательно, они принадлежат центрам, локализованным в позициях алюминия. Расчет показал, что указанные сигналы являются не наблюдавшимися в [4-6] из-за малой вероятности $\left(<10^{-2}\right.$ от вероятности разрешенных переходов) запрещенными переходами $(1 \leftrightarrow 3,2 \leftrightarrow 4$ и $1 \leftrightarrow 4)$ центров $\mathrm{Cr}^{3+}$.

Использование в fitting процедуре параметров спинового гамильтониана всех данных на рис. 1 и 6 привело к значениям табл. 4. Сравнение полученных

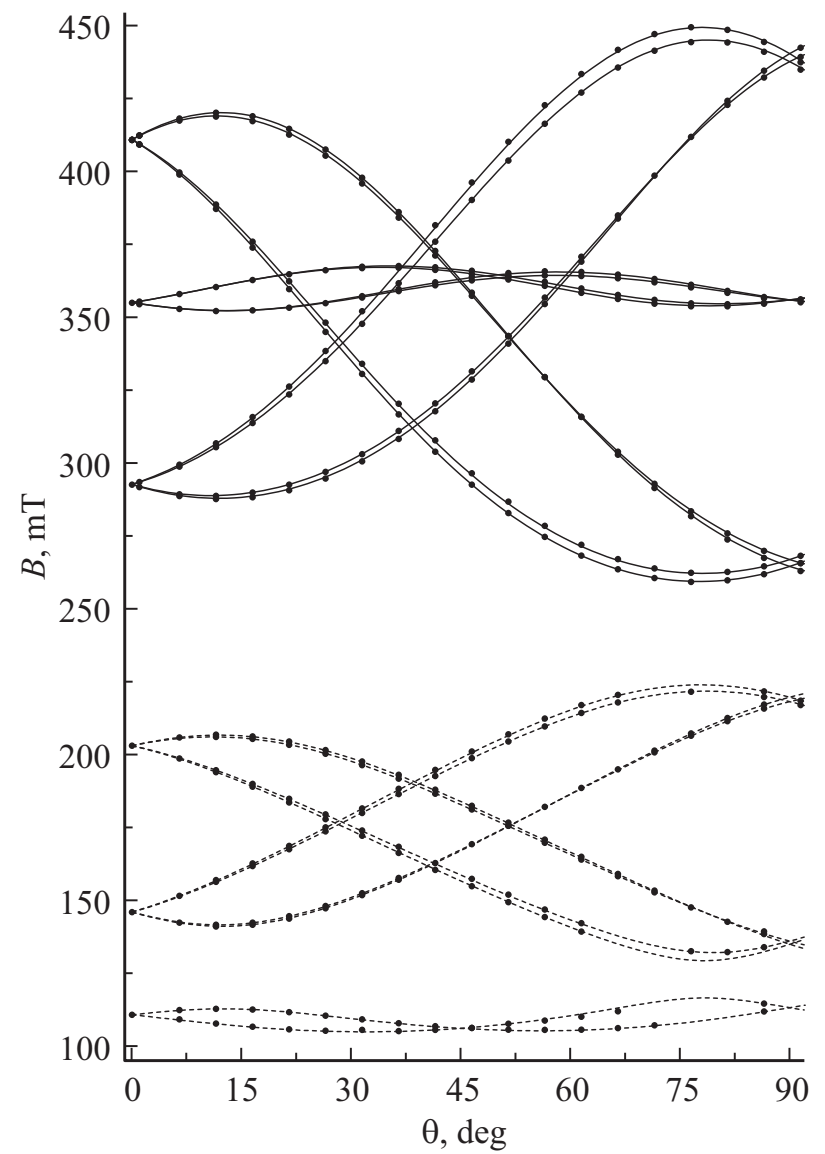

Рис. 6. Полярная угловая зависимость положений переходов $\mathrm{Cr}^{3+}$ вблизи плоскости zу (cb), точки - эксперимент, сплошные кривые - расчет для разрешенных переходов, штриховые - для запрещенных.
Таблица 4. Параметры триклинного спинового гамильтониана центров $\mathrm{Cr}^{3+}$ в $\mathrm{YAlO}_{3}$ в системе координат: $\mathbf{x}\|\mathbf{a}, \mathbf{y}\| \mathbf{b}, \mathbf{z} \| \mathbf{c}$ $\left(b_{n m}, c_{n m}\right.$ и $F(N)$ приведены в $\left.\mathrm{MHz}\right)$

\begin{tabular}{c|c}
\hline Параметры & $300 \mathrm{~K}$ \\
\hline$g_{x}$ & 1.9811 \\
$g_{y}$ & 1.9826 \\
$g_{z}$ & 1.9802 \\
$b_{20}$ & $824^{*}$ \\
$b_{21}$ & -1516 \\
$b_{22}$ & 1594 \\
$c_{21}$ & -1761 \\
$c_{22}$ & -335 \\
$F(N)$ & $8(417)$
\end{tabular}

Примечание. * знак $b_{20}$ не определялся.

параметров с результатами авторов [4-6] затруднено тем, что они определены в разных системах координат, однако можно сравнить не зависящие от системы координат величины начальных расщеплений основного состояния центров $\mathrm{Cr}^{3+}$ в $\mathrm{YAlO}_{3}$. Получены следующие значения: в данной работе $2828 \mathrm{MHz}(300 \mathrm{~K}), 2670 \mathrm{MHz}$ $(100-450 \mathrm{~K})$ в [4], $2838 \mathrm{MHz}(1.6 \mathrm{~K})$ в [5] и $3244 \mathrm{MHz}$ $(300 \mathrm{~K})$ в [6]. К сожалению, именно в работе [6], дающей для начального расщепления значение, заметно отличающееся от всех остальных, не указано какой экспериментальный материал использовался для определения параметров спинового гамильтониана, а также какова погрешность в описании эксперимента.

\section{4. Заключение}

В кристаллах алюмината иттрия, легированных изотопом европия 151 и кремнием, обнаружены ЭПР спектры именно центров ${ }^{151} \mathrm{Eu}^{2+}$, а также $\mathrm{Cr}^{3+}, \mathrm{Gd}^{3+}$ и $\mathrm{Mo}^{3+}$. Идентификация сигналов перечисленных центров проводилась с учетом их сверхтонкой структуры и литературных данных.

Уточнены параметры спинового гамильтониана центров $\mathrm{Gd}^{3+}$ и $\mathrm{Cr}^{3+}$ за счет использования большего, чем в ранних работах, количества переходов, а также более качественной ориентации образца.

Показано, что сигналы ионов $\mathrm{Mo}^{3+}(S=3 / 2)$, наблюдаемые авторами [2] и отнесенные ими к переходам двух неэквивалентных центров молибдена, являются двумя внутридублетными переходами одного центра ионов $\mathrm{Mo}^{3+}$, замещающих в $\mathrm{YAlO}_{3}$ ионы $\mathrm{Al}^{3+}$. Определены параметры тонкой структуры указанного центра.

\section{Список литературы}

[1] Г.Р. Асатрян, J. Rosa. ФТТ 44, 830 (2002).

[2] V.V. Laguta, A.M. Slipenyuk, J. Rosa, M. Nikl, A. Vedda, K. Nejezchleb, K. Blazek. Rad. Measurements 38, 735 (2004). 
[3] В.А. Важенин, А.П. Потапов, Г.Р. Асатрян, А.Г. Петросян, К.Л. Ованесян, А.В. Фокин. ФТТ 59, 1787 (2017).

[4] A. Pinto, N.Z. Sherman, M.J. Weber. J. Magn. Res. 6, 422 (1972).

[5] M. Yamaga, H. Takeuchi, T.P.J. Han, B. Henderson. J. Phys.: Condens. Matter. 5, 8097 (1993).

[6] I. Stefaniuk, C. Rudowicz, P. Gnutek, A. Suchocki. Appl. Magn. Reson. 36, 371 (2009).

[7] R.L. White, G.F. Herrmann, J.W. Carson, M. Mandel. Phys. Rev. A 136, 231 (1964).

[8] Н.М. Низамутдинов, Н.М. Хасанова, А.А. Галеев, Г.Р. Булка, В.М. Винокуров, В.А. Аккерман, Г.А. Ермаков. Кристаллография 34, 893 (1989).

[9] Н.М. Хасанова, Н.М. Низамутдинов, Г.Р. Булка, В.М. Винокуров, В.А. Аккерман, Г.А. Ермаков, А.А. Маркелов. Физика минералов и их синтетических аналогов. Изд-во Казанского ун-та. Казань (1988). С. 73.

[10] N.A. Toropov, I.A. Bondar, F.Ya. Galahov, H.S. Nikogosyan, N.V. Vinogradova. Изв. АН СССР. Сер. хим. 7, 1159 (1964).

[11] Х.С. Багдасаров, А.А. Каминский. Письма в ЖЭТФ 9, 501 (1969).

[12] Modern Crystallography / Ed. A.A. Chernov, E.I. Givargizov, Kh.S. Bagdasarov, V.A. Kuznetsov, L.N. Dem'yanets, A.N. Lobachev. In: B.K. Vainshtain. Nauka, M. (1980).

[13] A.G. Petrosyan. J. Crystal Growth 139, 372 (1994).

[14] S. Geller, E.A. Wood. Acta Cryst. 9, 563 (1956).

[15] R. Diehl, G. Brandt. Mater. Res. Bull. 10, 85 (1975).

[16] C.А. Альтшулер, Б.М. Козырев. Электронный парамагнитный резонанс соединений элементов промежуточных групп. Наука, М. (1972). С. 121.

[17] Э.Г. Шароян, О.С. Торосян, А.Г. Петросян, Э.А. Маркосян. Изв. АН Армянской ССР. Физика 12, 62 (1977).

[18] М.Л. Мейльман, М.И. Самойлович. Введение в спектроскопию ЭПР активированных монокристаллов. Атомиздат, М. (1977). С. 30.

[19] N.L. Ross, J. Zhao, R.J. Angel. J. Solid State Chem. 177, 1276 (2004).

[20] В.А. Важенин, А.П. Потапов, Г.С. Шакуров, А.В. Фокин, М.Ю. Артёмов, В.А. Исаев. ФТТ 60, 2003 (2018).

[21] В.А. Важенин, А.П. Потапов, В.Б. Гусева, М.Ю. Артёмов. ФTT 52, 480 (2010).

Редактор К.В. Емцев 